\title{
An Inflection Point Approach for Advertising Bid Optimization
}

\author{
Deguang Kong, Konstantin Shmakov and Jian Yang \\ Yahoo Research, Oath, 701 1st Ave, Sunnyvale, California, 94086 \\ \{dkong,kshmakov,jianyang\}@oath.com
}

\begin{abstract}
In online advertising, a common objective for advertisers is to get the maximum returns on investment given the budget. On one hand, if the bid is too high, the advertiser pays more money than he should pay for the same number of clicks. On the other hand, it the bid is too low, the advertiser cannot win in auctions and therefore it loses the opportunity. A challenging problem is how to recommend the bid to achieve the maximum values for advertisers. In this paper, we present an inflection point approach for bid recommendation from discovering the bid price of click(bid $)^{1}$ function at which the function changes from significant increase (i.e. concave downward) to slow increase (convex upward). We derive the optimal solution using history sparse and noisy observations given the budget limit. In real word advertising campaign evaluations, the proposed bid recommendation scenario brings in $15.37 \%$ bid increase and $30.24 \%$ click increase over the baselines.
\end{abstract}

\section{CCS CONCEPTS}

\section{- Information systems $\rightarrow$ Computational advertising;}

\section{KEYWORDS}

Advertising; Optimization; Bidding

\section{ACM Reference Format:}

Deguang Kong, Konstantin Shmakov and Jian Yang. 2018. An Inflection Point Approach for Advertising Bid Optimization. In WWW'18: The 2018 Web Conference Companion, April 23-27, 2018, Lyon, France. ACM, New York, NY, USA, 2 pages. https://doi.org/10.1145/3184558.3186944

\section{INTRODUCTION}

Online advertising brings in a lot of revenues to major internet companies based on generalized second-price (GSP) [1] using algorithmic/dynamic pricing [5] with particular optimization optimization goals, such as CPA goal [4], portfolio optimization [3], etc. In online advertising, one major complaint of advertisers is: "Half the money I spend on advertising is wasted; the trouble is I don't know which half ${ }^{2}$." As an advertiser, one always needs to consider the spending question, such as "am I spending enough on advertising?" and "am I spending too much on advertising" ? One key observation in ad market is: advertisers' return on investment has non-linearity

\footnotetext{
${ }^{1}$ This is a function with bid price as the argument and the number of clicks as the function value.

${ }^{2}$ The original words are from John Wanamaker, who is a proponent of advertising and a pioneer in marketing.
}

This paper is published under the Creative Commons Attribution 4.0 International (CC BY 4.0) license. Authors reserve their rights to disseminate the work on their personal and corporate Web sites with the appropriate attribution.

WWW'18, April 23-27, 2018, Lyon, France

() 2018 IW3C2 (International World Wide Web Conference Committee), published under Creative Commons CC BY 4.0 License.

ACM ISBN 978-1-4503-5640-4/18/04.

https://doi.org/10.1145/3184558.3186944 characteristics due to diminishing returns after the advertiser's bid reaches to a certain point which in fact leads to saturation effect. For example, if the advertiser bids at $\$ 3.60$, it may get around $450 \mathrm{k}$ clicks; however, if the advertiser increases the bid to $\$ 6.00$, the true clicks are only $600 \mathrm{k}$ which is far less than two thirds more than the clicks at $\$ 3.60$. The diminishing return is known as "saturation effect" due to the reach of the limit of click where even higher bidder can have very minor incremental effect.

In this paper, we design an inflection point based strategy to achieve the maximum return increase using history ad campaign observations in marketplace, which we formalize as an optimization problem with the budget limit constraint. Our model is originated from the concept of inflection point, which aims to discover the significant change (a.k.a a turning point) in a function curve at which the sign of the curvature changes. The bid recommendation using inflection point demonstrates good performance and has graduated into production.

\section{METHODOLOGY}

For an advertiser, in order to maximize the returns for the amount of spend, the bid recommendation method aims to recommend the bid price to meet this criterion. There are many ways to define the returns over the amount of spend, such as the revenue increase over spend increase, click increase over cost increase, etc. In the context of this paper, we aims to find the best bid price which provides the maximum click increase $d$ Click over the cost increase $d$ Cost for an advertiser, which is formulated below:

$$
\max _{\text {bid }} \frac{d \text { Click(bid) }}{d \text { eCPM_Cost(bid) }} \text { s.t. Spend(bid) } \leq \text { budget, }
$$

where Click(bid) is the estimated clicks at bid price (cost-per-click), eCPM_Cost (bid) is the effective-cost-per-mille (eCPM cost) at bid price, Spend(bid) is the estimated money spent at bid price (costper-click), budget is the available number of money for the campaign, $d($.$) is the first-order difference that reflects the changes in$ quantities, i.e.,

$$
d \mathrm{~h}(\text { bid }):=\Delta \mathrm{h}(\text { bid })=\mathrm{h}(\text { bid }+\Delta \text { bid })-\mathrm{h}(\text { bid })
$$

and function $h$ could be the number of click, the amount of cost, and the amount of spend, etc. Given the advertiser history performance, we will have the estimated number of clicks, the amount of cost and the amount of spend, i.e.,

$$
\begin{aligned}
& \text { (a) } \operatorname{Click}(\text { bid })=\text { Impression } \times \text { Winrate }(\text { bid }) \times C T R \text {; } \\
& \text { (b) } \quad \operatorname{Spend}(\text { bid })=\operatorname{Click}(\text { bid }) \times \frac{\text { eCPM_cost(bid) }}{1000 \times C T R} ;
\end{aligned}
$$

where impression is the total number of impressions the advertiser can win from the supply side (offered by publishes based on advertiser profile), CTR is the average click-through-rate, Click(bid) is the number of winning clicks at price bid, and winrate(bid) is the winning rate at price $b i d$. 
Optimization Notice that number of Click and eCPM_Cost are both functions of bid price, and therefore, Click can be modeled as a function of eCPM_Cost, i.e.,

$$
\text { Click :=h(eCPM_Cost), }
$$

where $h$ is a function that maps the eCPM_Cost to the number of Click. Then we have,

$$
\begin{gathered}
\frac{\Delta_{\text {Click }}}{\Delta_{\text {eCPM_cost }}}=\frac{d h}{d e C P M \_C o s t} \\
:=h^{\prime}(\text { eCPM_Cost }),
\end{gathered}
$$

where $h^{\prime}$ is the first-order derivative of function $h($.$) . Therefore$ Eq.(1) is transformed to solving the following problem,

$$
\begin{aligned}
& \max _{\mathrm{eCPM}} \text { cost } h^{\prime}(\mathrm{eCPM} \text { _Cost }), \\
& \text { s.t. } \quad \text { Spend(eCPM_cost }) \leq \text { budget. }
\end{aligned}
$$

In practice, we need to fit the observations using $h($.$) function with$ parameter learning and derive the corresponding optimal solutions. Then the optimal eCPM_cost is given by:

$$
\text { eCPM_cost }{ }^{*}=\operatorname{argmax}_{x} h^{\prime}(x) \text {. }
$$

We can obtain the optimal solution by setting the second order of derive to zero, i.e, $\frac{d^{2} h}{d x^{2}}=0$. This is not done. We need to further check whether the spend exceeds the budget or not. In particular, if

$$
\text { Spend }\left(\mathrm{eCPM} \text { cost }^{*}\right) \leq \text { budget, }
$$

then eCPM_cost ${ }^{*}=x^{*}$ is the optimal solution. Otherwise, if the spend already exceeds the budget, we need to decrease eCPM_cost ${ }^{\star}$ until spend is within the budget because $h($.$) is a non-decreasing$ function. In particular, we use a binary search algorithm to search for the highest eCPM_cost* with the amount of spend at most equal to the budget, i.e.,

$$
\text { eCPM_cost }{ }^{*}=\text { binary_search }\left(0, x^{*}, \text { budget }\right) \text {. }
$$

Given the best eCPM_cost, one can easily obtain CPC bid price via transformation using click-through-rate (CTR), i.e.,

$$
\text { CPC_cost }(\text { bid })=\frac{\text { eCPM_cost }(\text { bid })}{1000 \times \mathrm{CTR}} \text {. }
$$

\section{EXPERIMENT}

We apply the proposed algorithm for real-world advertising campaigns. All experiments are performed on 120,796 ad campaign profiles. Fig.1 plots an example of number of Click vs. eCPM_cost curves using CTR prediction [2] and the corresponding derivatives $\left(\frac{d \text { Click }}{d \text { eCPM_cost }}\right)$. In left panel, we fit the observations. In the right panel, the maximum derivate (i.e., the inflection point) is computed and denoted in red circle in the curve of derivative (in dashed blue). "Max $90 \%$ " is the point that achieves the $90 \%$ of maximum derivative but goes beyond the inflection point, where the bid price bid ${ }_{I P 90}^{*}$ that achieves $90 \%$ of $\frac{d \text { Click }}{d \text { eCPM_cost }}$ but goes beyond the inflection point.

In experiments, we can easily find the inflection point using our method. Our method gives the optimal solution that achieves the maximum derivative no matter whether there exist noisy or missing values in observations. Essentially, the outliers and missing values are smoothed out, making the result more robust and reliable. In order to see how the performance improves for real-world ad campaigns, we apply A/B test to the ad traffic using two scenarios
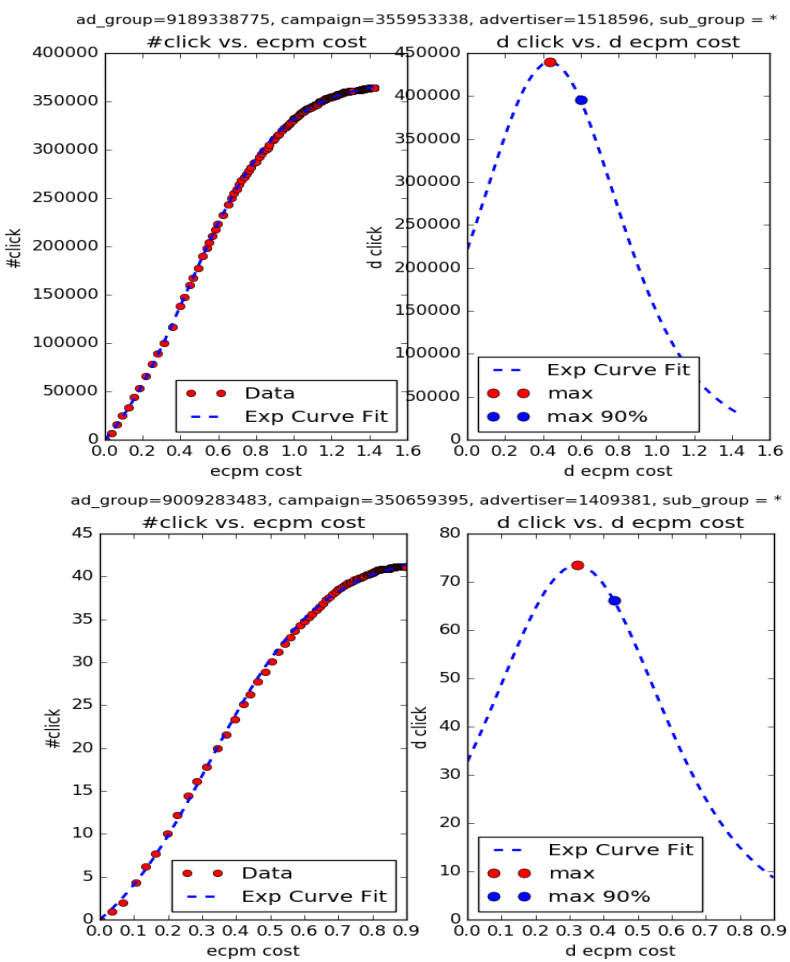

Figure 1: Number of Click vs. eCPM_cost curves and the corresponding derivatives $\left(\frac{d \text { Click }}{d \text { eCPM cost }}\right)$ on ad campaigns. Left panel: curves to fit the observations. Right panel: the maximum derivate (i.e., the inflection point) is obtained in red circle in derivative curve (in dashed blue). "Max $90 \%$ " is the point that achieves $90 \%$ of maximum derivatives but goes beyond the inflective point.

(1) without any bid optimization;

(2) adoption of the infection point as the recommended bid.

We have $\mathbf{+ 1 5 . 3 7 \%}$ in bid increase and $\mathbf{+ 3 0 . 2 4 \%}$ click increase compared to bidding without any optimization.

\section{CONCLUSION}

This paper presents a novel way for bid recommendation using inflection point in providing the optimal bid solution based on maximizing the click increase over the cost increase. Rigorous algorithm analysis and extensive experiments on real-world advertising campaigns demonstrate the effectiveness of our method over baselines.

\section{REFERENCES}

[1] Benjamin Edelman, Michael Ostrovsky, and Michael Schwarz. 2007. Internet Advertising and the Generalized Second-Price Auction: Selling Billions of Dollars Worth of Keywords. American Economic Review 97, 1 (March 2007), 242-259.

[2] Hongchang Gao, Deguang Kong, Miao Lu, Xiao Bai, and Jian Yang. 2018. ContextAware Attention Convolutional Neural Network for Advertiser-Level CTR Forecasting. In WWW'2018. ACM, to appear.

[3] Deguang Kong, Xiannian Fan, Konstantin Shmakov, and Jian Yang. 2018. A combinational optimization approach for advertising budget allocation. In WWW'2018. ACM, to appear.

[4] Deguang Kong, Konstantin Shmakov, and Jian Yang. 2018. Demystifying Advertising Campaign for CPA Goal Optimization. In WWW'2018. ACM, to appear.

[5] Weinan Zhang, Shuai Yuan, and Jun Wang. 2014. Optimal Real-time Bidding for Display Advertising. In KDD '14. ACM, 1077-1086. 